\title{
Magnetic and Structure Properties of Fe/Pd Multilayers Studied by Magnetic X-Ray Dichroism and X-Ray Absorption Spectroscopy
}

\author{
S. M. Mini ${ }^{*}, \nexists, \nexists$, E. E. Fullerton ${ }^{\neq}$, A. Fontaine ${ }^{*}$, C. H. Sowers ${ }^{\neq}$, A. S. Bommannavar ${ }^{\dagger}$, A. Traversel, \\ F. Baudelet $\S$, and S. Pizzini*,
}

*Laboratoire pour l'Utilisation du Rayonnement Electromagnetique (LURE), Bât. 209D, Centre Universitaire Paris-Sud, 91405 Orsay, France

₹Materials Science Division, Argonne National Laboratory, Argonne, IL 60439

tEXXON, National Synchrotron Light Source, Brookhaven National Lab., Upton, NY 11973

ICentre de Spectrometrie Nucl. et de Spectrometrie de Masse, CNRS, Orsay, France

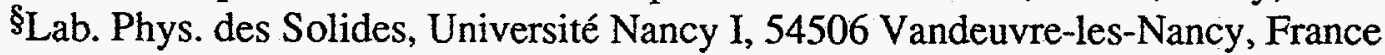

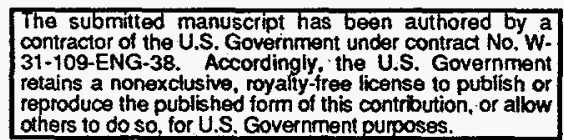

December, 1994

$/ \mathrm{sm}$

\section{DISCLAIMER}

\begin{abstract}
This report was prepared as an account of work sponsored by an agency of the United States Government. Neither the United States Government nor any agency thereof, nor any of their employees, makes any warranty, express or implied, or assumes any legal liability or responsibility for the accuracy, completeness, or usefulness of any information, apparatus, product, or process disclosed, or represents that its use would not infringe privately owned rights. Reference herein to any specific commercial product, process, or service by trade name, trademark, manufacturer, or otherwise does not necessarily constitute or imply its endorsement, recommendation, or favoring by the United States Government or any agency thereof. The views and opinions of authors expressed herein do not necessarily state or reflect those of the United States Government or any agency thereof.
\end{abstract}

\section{MASTER}

To be Published in the Proceedings of the Materials Research Society Fall ' 94 Meeting, Boston, MA $11 / 28-12 / 2 / 94$

This work is supported by the Division of Materials Sciences, Office of Basic Energy Sciences of DOE, under contract No. W-31-109-ENG-38.

¥Permanent address: Dept. of Physics, Northern Illinois University, DeKalb, IL 60115 and ₹ Materials Science Division, Argonne National Laboratory, Argonne, IL 60439 


\section{DISCLAIMER}

Portions of this document may be illegible in electronic image products. Images are produced from the best available original document. 
MAGNETIC AND STRUCTURAL PROPERTIES OF Fe/Pd MULTILAYERS STUDIED BY MAGNETIC X-RAY DICHROISM AND X-RAY ABSORPTION SPECTROSCOPY

S.M. Mini*¥, Eric E. Fullerton**, A. Fontaine*, C.H. Sowers**, A.S. Bommannavar ${ }^{*}$, A. Traversel, F. Baudelet $\S$, and S. Pizzini*

* Laboratoire pour l'Utilisation du Rayonnement Electromagnétique (LURE), Bât. 209D, Centre Universitaire Paris-Sud, 91405 Orsay, France

** Materials Science Division, Argonne National Laboratory, Argonne, IL 60439

† EXXON, National Synchrotron Light Source, Brookhaven National Laboratory, NY 11973

If Centre de Spectrometrie Nucl. et de Spectrometrie de Masse, CNRS, Orsay, France

$\S$ Lab. Phys. des Solides, Université Nancy I, 54506 Vandeuvre-les-Nancy, France

\section{ABSTRACT}

The results of magnetic circular x-ray dichroism (MCXD) measurements and extended $\mathrm{x}$-ray absorption fine structure measurements (EXAFS) of the Fe K-edges of textured $\mathrm{Fe}(110) / \mathrm{Pd}(111)$ multilayers are reported. The EXAFS results indicates that the iron in the system goes from bcc to a more densely packed system as the thickness of the iron layer is decreased. The magnetic properties were measured by SQUID magnetometry from $5-350 \mathrm{~K}$. For all the samples, the saturation magnetization was significantly enhanced over the bulk values indicating the interface Pd atoms are polarized by the Fe layer. The enhancement corresponds to a moment of $\approx 2.5 \mu_{\mathrm{B}}$ per interface Pd atom.

\section{INTRODUCTION}

Due to their unique magnetic properties $\mathrm{Pd} /$ transition metal systems have been of interest for some time. In fact, reports that contact between $\mathrm{Pd}$ and $\mathrm{Fe}$ in dilute $\mathrm{PdFe}$ alloys produce large magnetic polarization 'giant moments' date back to 1965 [1]. Recently, similar polarization effects of Pd have been found for textured and epitaxial Fe/Pd multilayers [2-4]. Pd atoms at an $\mathrm{Fe} / \mathrm{Pd}$ interface are strongly polarized by the $\mathrm{Fe}$ atoms. This polarization is often observed by an enhanced magnetic moments in the $\mathrm{Fe} / \mathrm{Pd}$ multilayers and strong ferromagnetic interlayer coupling for thin Pd layers. The magnetic properties of the $\mathrm{Fe} / \mathrm{Pd}$ multilayer systems are strongly dependent upon the structural characteristics of the samples. In most sputtered superlattices, the layers typically grow normal to the densest planes resulting in textured $\mathrm{Fe}(110) / \mathrm{Pd}(111)$ superlattices. The in-plane lattice mismatch is $4.2 \%$ along the $\mathrm{Fe}<100>$ and $17 \%$ along the $\mathrm{Fe}$ $<110>$. Epitaxial superlattices are typically $\mathrm{Fe}(001) / \mathrm{Pd}(001)$ with a $4.2 \%$ in-plane lattice mismatch. The polarization and interlayer coupling have been found to depend on a number of structural properties including interfacial roughness and interdiffusion, and tetragonal distortions of the Pd layers [2].

$X$-ray absorption near edge structure (XANES) and extended $x$-ray absorption fine structure (EXAFS), are local probes and provide information about the electronic and structural properties respectively of the different elements in the system. For further discussion of these techniques see Hayes and Boyce or Heald [5]. 
Magnetic circular x-ray dichroism (MCXD) is a probe of the local magnetic properties of ferromagnetic or ferrimagentic materials. Since it is a local probe it provides a means of discerning the magnetic contributions of each element (Fe and Pd) separately. The advantages of magnetic $x$-ray absorption measurements are atom selectivity, the magnetic signal can be probed independently for each chemical species in the sample, and that the measurement obeys the electric dipole selection rules which determine the symmetry of the final state.

MCXD is the difference between the absorption of circularly polarized X-rays by a material magnetized by a magnetic field applied respectively in the direction antiparallel and parallel to the propagation vector of the $\mathrm{x}$-ray beam. The MCXD signal is usually defined as the absorption difference normalized to the total absorption, $I_{M C X D}=\left(\mu^{-}-\mu^{+}\right) /\left(\mu^{-}+\mu^{+}\right)$where $\mu^{-}(\mu+)$ is the absorption coefficient of the sample when the magnetic field is applied antiparallel (parallel) to the propagation direction of an x-ray beam with right circular polarization.

The first MCXD measurements in the $\mathrm{x}$-ray regime were carried out by van der Laan, et al. with linearly polarized soft x-rays [6] and by G. Schütz et al. with circularly polarized hard $\mathrm{x}$-rays [7]. While magnetic dichroism signals deriving from localized electronic states, as in the 4f states for $M_{I V}, v$ edges of rare earth elements, can be fully described using the atomic multiplet formalism [8] the interpretation of the MCXD at the K-edge of transition metals, which probe the conduction band magnetism, is not well understood [9].

The K-edge MCXD signal of transition metals is in general very weak $\left(10^{-3}\right)$ since it is induced by the spin-orbit interaction in the $4 \mathrm{p}$ band. Nevertheless, K-edge $\mathrm{X}$-ray absorption spectroscopy presents some important advantages for the study of magnetic multilayers: (1) it probes directly the conduction electrons which are expected to mediate the magnetic interaction; (2) it avoids the limitation brought by soft $\mathrm{x}$-rays whose sensitivity is restricted to a few layers close to the surface.

The various shapes of the K-edge MCXD spectra may be qualitatively correlated with weak (in the case of $\mathrm{Fe}$ ) or strong ferromagnetic nature of the material. The MCXD spectrum of pure iron exhibits a double peaked structure, with a positive and negative peak covering a total energy range of about $17 \mathrm{eV}$ around the absorption edge. Fe is a weak ferromagnetic material (its Fermi level crosses both the majority and minority spin d-bands). It has been suggested that the positive peak could be related to the dominant spin-up d-like density of states close to the Fermi level, specific to $\mathrm{Fe}$, while the negative peak observed at higher energies would be related to the spin-down density of states. Multiple scattering calculations [9] partly support this qualitative correlation as they show that the positive peak observed in Fe K edge MCXD spectrum of pure Fe is related to the $\mathrm{d}$ component of the photoelectron scattered by the spin-orbit of the neighboring atoms.

\section{SAMPLE PREPARATION AND EXPERIMENT}

A series of $\mathrm{Fe} / \mathrm{Pd}$ multilayers were synthesized by dc magnetron sputtering onto ambient temperature kapton substrates. An initial layer of $100 \AA$ of $\mathrm{Cr}$ was deposited prior to the deposition of the multilayer. The multilayers were of the form $\mathrm{Cr}(100 \AA) /[\mathrm{Fe}(\mathrm{x}) / \mathrm{Pd}(\mathrm{y})]_{30}$ where $x$ and y equal $20 \AA, 20 \AA ; 20 \AA, 10 \AA ; 20 \AA, 4 \AA ; 10 \AA, 20 \AA ;$ and $4 \AA, 20 \AA$. The x-rays were measured using $\mathrm{Cu} \mathrm{K}_{\alpha}$ radiation. The magnetic properties were measured by SQUID magnetometry from $5-350 \mathrm{~K}$. EXAFS spectra at the Fe K-edge were measured in electron yield mode at beamline D41 at LURE, using a Si (331) channel cut monochromator.

MCXD measurements were carried out on the energy dispersive $x$-ray absorption beamline of the positron injected storage ring DCI at the French synchrotron radiation facility LURE. The exceptionally long lifetime $(\approx 100$ hours) and good spatial stability of the source are necessary for such low signal measurements. Right circularly polarized photons (polarization rate approximately $80 \%$ ) were selected by positioning a $1 \mathrm{~mm}$ wide slit $3 \mathrm{mrad}$ below the synchrotron orbit plane. The $\mathrm{x}$-ray absorption spectra were measured using a Si (111) curved polychromator, focused $80 \mathrm{~cm}$ away, in the gap between the poles of an electromagnet. Higher harmonics were rejected by a zerodur mirror positioned before a photodiode array detector. The absorption spectra were measured, in transmission geometry, in a magnetic field applied alternatively anti- 
parallel $\left(\mu^{+}\right)$and parallel $\left(\mu^{-}\right)$to the propagation direction of the $x$-ray beam. The magnetic field direction was switched typically 500 times between the two positions and the total acquisition time was about eight hours. For the most dilute sample, the total acquisition time was three days. For multilayers, a glancing angle geometry was used to enhance the volume being probed. In this geometry the magnetic field was approximately in the plane of the layers. The poles of the electromagnet were kept farther apart and the magnetic field applied to the samples was $0.4 T$. In all cases, the applied magnetic field was sufficient to obtain magnetic saturation.

The absorption spectra of the multilayers were measured at the Fe-K edge (7112 eV). The difference spectra $\left(\mu^{+}-\mu^{-}\right)$were normalized by division by a constant value, the absorption edge step height. The normalized difference spectrum will be referred to as the MCXD signal. In all of the samples, the amplitude was on the order of $1-2 \times 10^{-3}$. The origin of the energy scale was chosen as the steepest point in the rising edge $(7112 \mathrm{eV})$.

\section{RESULTS AND DISCUSSION}

All the samples were textured $\mathrm{Fe}(110) / \mathrm{Pd}(111)$ as shown by the $\mathrm{x}$-ray diffraction spectra in Fig. 1. The fit to the multilayer peak intensities gives an average $\mathrm{Fe}$ and $\mathrm{Pd}$ lattice spacings of $2.07 \AA$ and $2.255 \AA$, respectively for the $[\mathrm{Fe}(20 \AA) / \mathrm{Pd}(20 \AA)] 30$ samples [10]. These values are expanded over the bulk materials $(2.027 \AA$ and $2.246 \AA)$. Such expansions are commonly observed in lattice-mismatched metallic superlattice and has been attributed to defects arising from the interfaces.

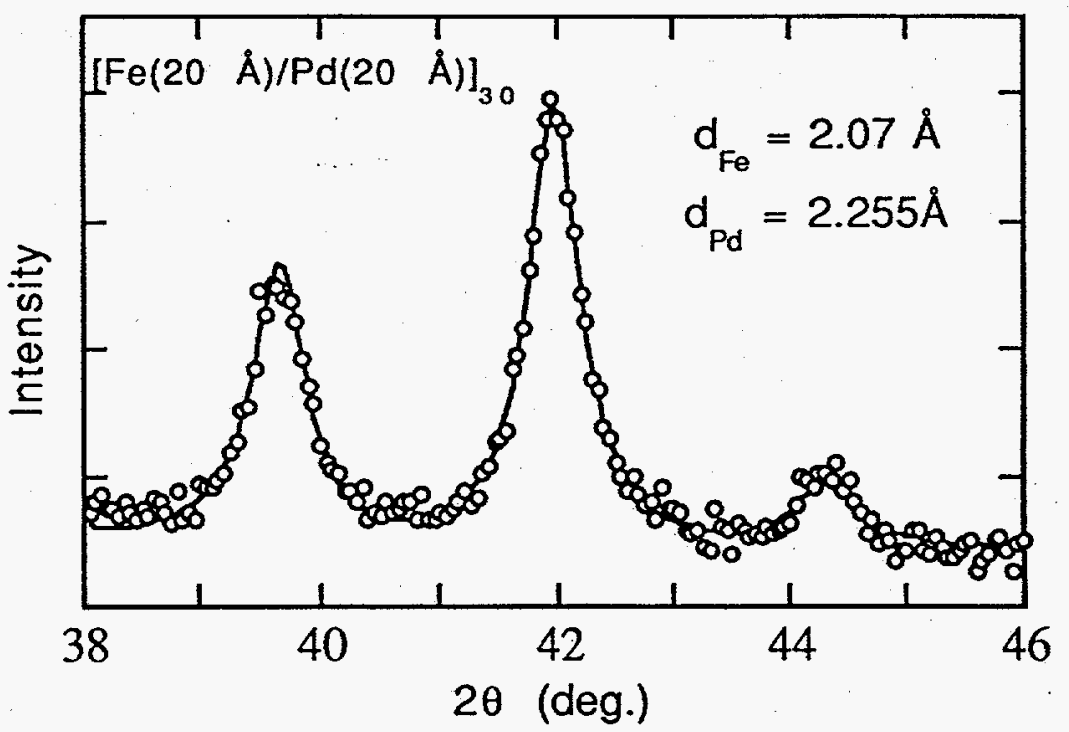

Figure 1. X-ray diffraction spectrum of an $\left[\mathrm{Fe}(20 \AA) / \mathrm{Pd}(20 \AA]_{30}\right.$ multilayer The fit to the multilayer peak intensities gives an average Fe and Pd lattice spacings of $2.07 \AA$ and $2.255 \AA$ respectively.

For all the samples, the saturation magnetization at low temperatures was significantly enhanced over the value expected for bulk Fe indicating that the interface Pd atoms are polarized by the Fe layer, as seen in Fig. 2 . The enhancement, measured at $5 \mathrm{~K}$, corresponds to a moment of $\approx 2.5 \mu_{\mathrm{B}}$ per interface Pd atom. This value is consistent with the work of Childress et al. [3] for MBE grown Fe/Pd(001) superlattices, but much higher than that for MBE grown Fe/Pd(001) bilayers or theoretical calculations [2]. This difference can be understood from roughness and/or interdiffusion at the $\mathrm{Fe} / \mathrm{Pd}$ interface which results in a larger number of $\mathrm{Pd}$ atoms in proximity with $\mathrm{Fe}$ atoms. The polarized moment measured at $4.2 \mathrm{~K}$ for fixed Fe thickness is nearly 
independent of Pd thickness, indicating a majority of the polarized moment is localized at the $\mathrm{Fe} / \mathrm{Pd}$ interface. However, there is a systematic change in the temperature dependence of the saturation magnetization with changes in both the Fe and Pd thicknesses. For fixed Fe thickness, the saturation magnetization is more strongly temperature dependent for thicker Pd layers. Similarly, for fixed Pd thickness (Fig. 2) the magnetization is more strongly temperature dependent for thinner Fe thicknesses. However, for all samples the M-vs.-T data is well represented by the $\mathrm{T}^{3 / 2}$-behavior expected for magnons in a 3-D ferromagnetic. This suggests that the even for the $\mathrm{Fe}(4 \AA) / \mathrm{Pd}(20 \AA)$ sample, the Fe layers are ferromagnetically coupled through the $\mathrm{Pd}$ layers giving rise to the $3-\mathrm{D}$ temperature behavior.

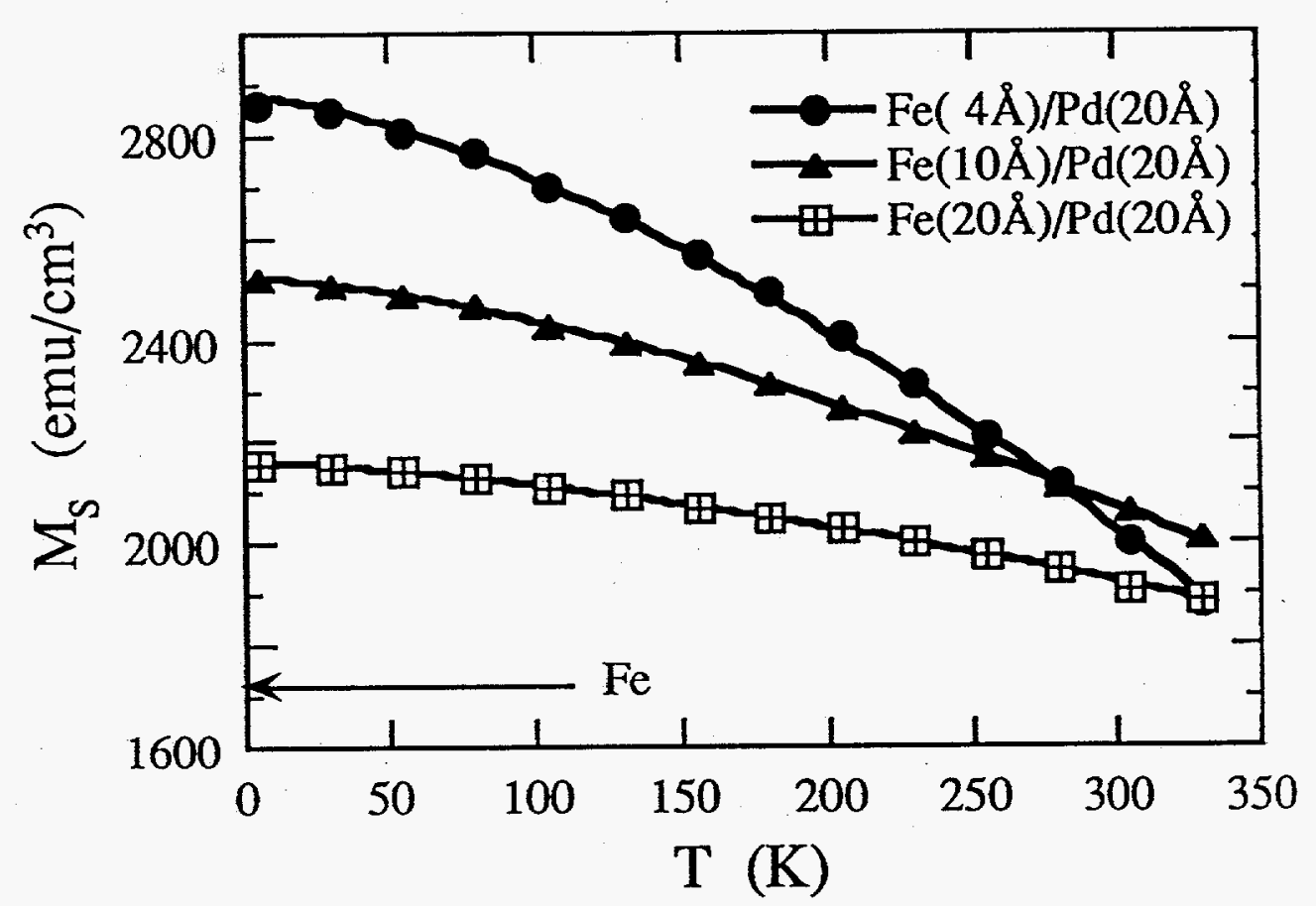

Figure 2. SQUID magnetometry results for the saturation magnetization $\left(\mathrm{M}_{\mathrm{S}}\right.$ per cm${ }^{3}$ of $\mathrm{Fe}$ ) vs. Temperature for $\mathrm{Fe}(\mathrm{x}) / \mathrm{Pd}(20 \AA)$ multilayers. $\mathrm{M}_{\mathrm{S}}$ for the multilayers is enhanced over the bulk $\mathrm{Fe}$ value indicating that the interface $\mathrm{Pd}$ atoms are polarized by the Fe layer. The solid lines are fits to the equation $M_{S}(T)=M_{S}(0)\left[1-\alpha T^{3 / 2}\right]$.

The near edge of the $\mathrm{x}$-ray absorption measurements for three of the multilayers with the Pd layer held constant are shown in Fig. 3. The edge of an Fe foil which has the bulk bcc $(2.87 \AA)$ structure is shown for comparison. The change in the shape of the edges indicate a change in structure from bcc-Fe to, most likely, fcc for the smallest Fe layers. The Fe K-edge EXAFS, as shown in Fig. 4 (a) and (b), indicate that the $\mathrm{Fe}(20 \AA) / \mathrm{Pd}(\mathrm{y})$ multilayers are similar to the EXAFS of the Fe foil, with the amplitude of the first peak decreasing with increasing Pd layer thickness. For the Fe foil, the first peak is typically fit with a coordination number, $\mathrm{CN}_{1}=8$, for $r_{1}=2.48 \AA$ and $\mathrm{CN}_{2}=6$ for $\mathrm{r}_{2}=2.866 \AA$. For the second peak, $\mathrm{CN}_{3}=12$ and $\mathrm{r}_{3}=4.05 \AA$. In Fig. 4(a), the shape and position of the first two peaks for the multilayers follow that of the iron foil, indicating the $\mathrm{Fe}$ in these systems behaves in a bulk-like manner. However, for $\mathrm{Fe}(10 \AA) / \mathrm{Pd}(20 \AA)$ as shown in Fig. 4(b), the first peak shows a slight shift whereas for $\mathrm{Fe}(4 \AA) / \mathrm{Pd}(20 \AA)$, the first peak splits into two, indicating a loss of the Fe bulk structure. This is in agreement with the Fe K-edge XANES of these samples, in that the shape of the near edge indicates a change in the Fe structure from bec for thin Fe layers. A similar transition was observed in Fe/Cu multilayers where the Fe transforms from bcc to fcc at a critical thickness [11]. The structural change in the Fe layer may also result from formation of an ordered FePd compound at the interface which can have an fcc or fct structure. 


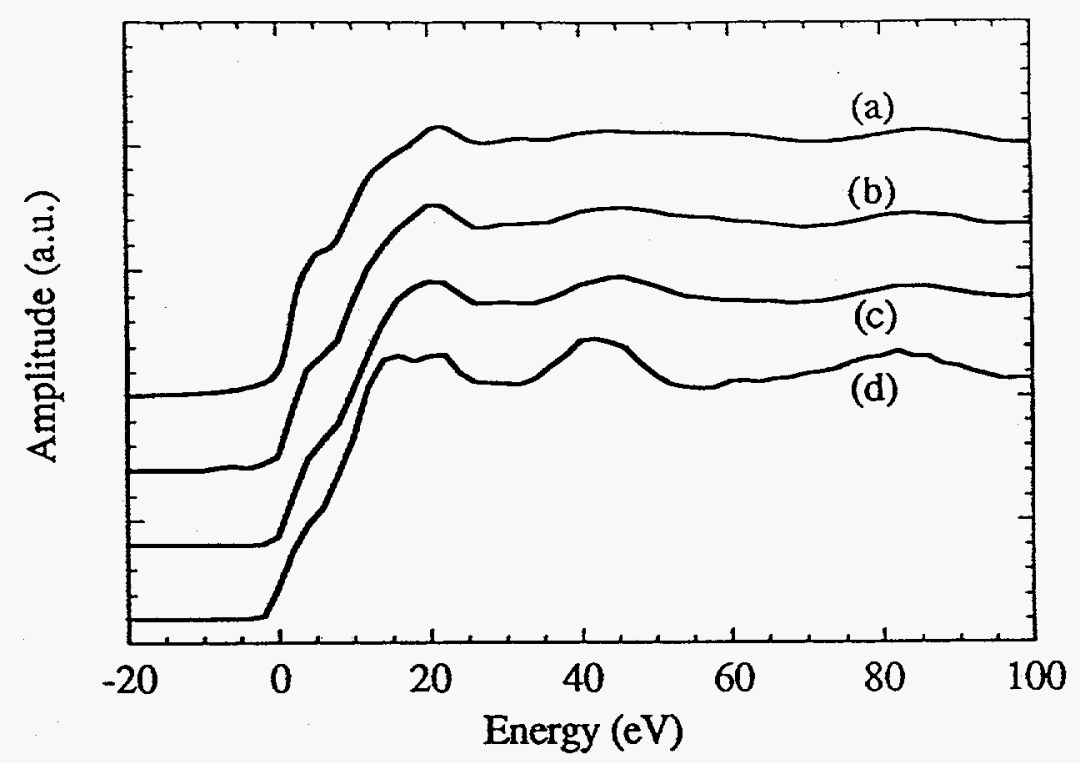

Figure 3. The Fe $\mathrm{K}$ near edge $\mathrm{x}$-ray absorption spectra for some multilayers are compared with the Fe K-edge spectra of pure bcc Fe. (a) Fe foil,

(b) $\mathrm{Fe}(20 \AA) / \mathrm{Pd}(20 \AA)$, (c) $\mathrm{Fe}(10 \AA) / \mathrm{Pd}(20 \AA)$, and (d) $\mathrm{Fe}(4 \AA) / \mathrm{Pd}(20 \AA)$.
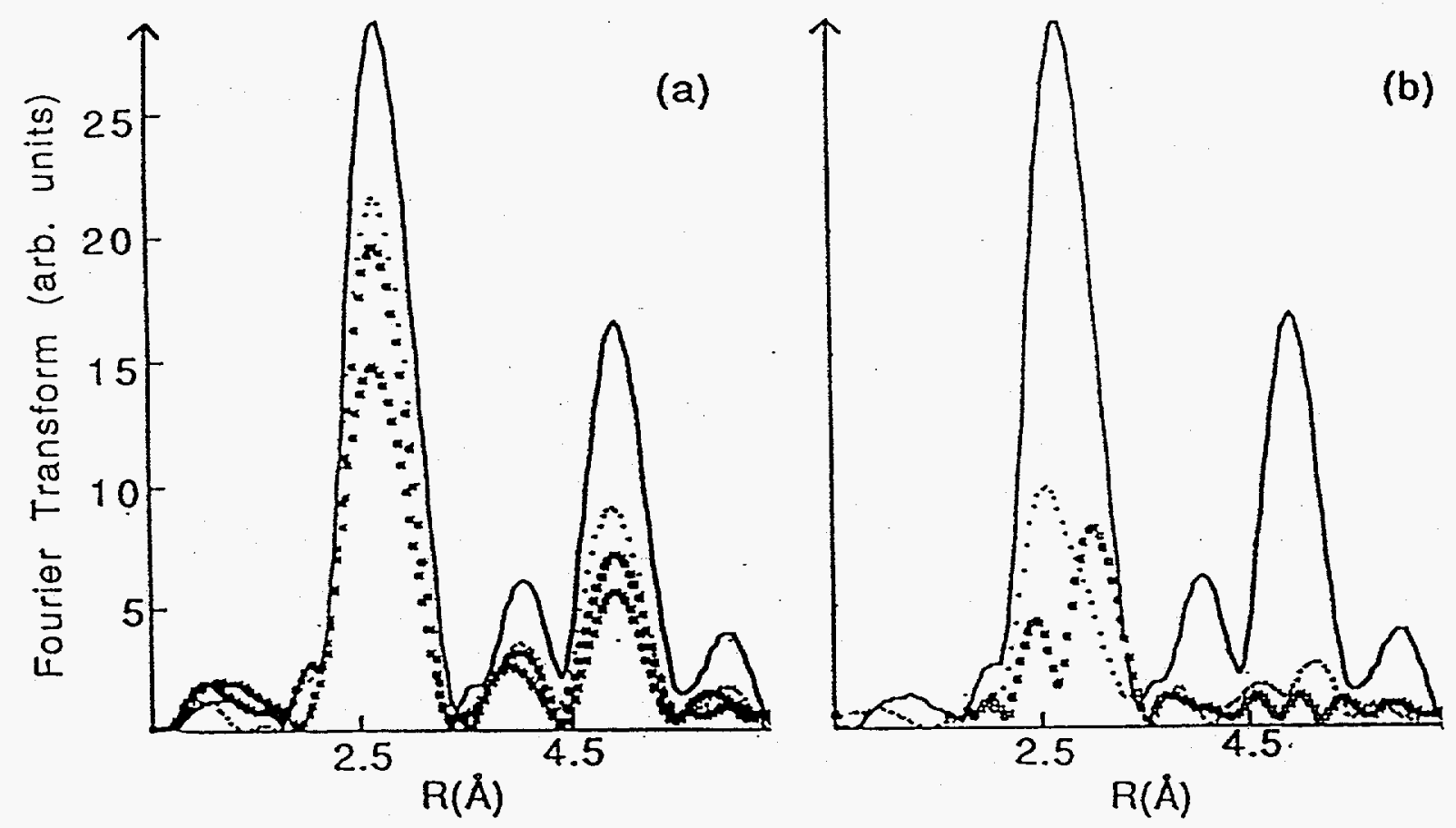

Figure 4. The Fourier transforms (FT) of Fe K-edge EXAFS spectra of multilayers are compared with the $\mathrm{Fe}$ of the bulk Fe at the Fe K-edge $\left(3 \rightarrow 13 \AA^{-1}\right)$. (a) Fe foil ( - ), $\mathrm{Fe}(20 \AA) / \mathrm{Pd}(4 \AA)(\cdots .),. \mathrm{Fe}(20 \AA) / \mathrm{Pd}(10 \AA)(\cdots \cdots)$, and $\mathrm{Fe}(20 \AA) / \mathrm{Pd}(20 \AA)(* * * * *)$; (b) $\mathrm{Fe}$ foil $(-), \mathrm{Fe}(10 \AA) / \mathrm{Pd}(20 \AA)(\cdots .$.$) , and \mathrm{Fe}(4 \AA) / \mathrm{Pd}(10 \AA)(\cdots \cdot$. 


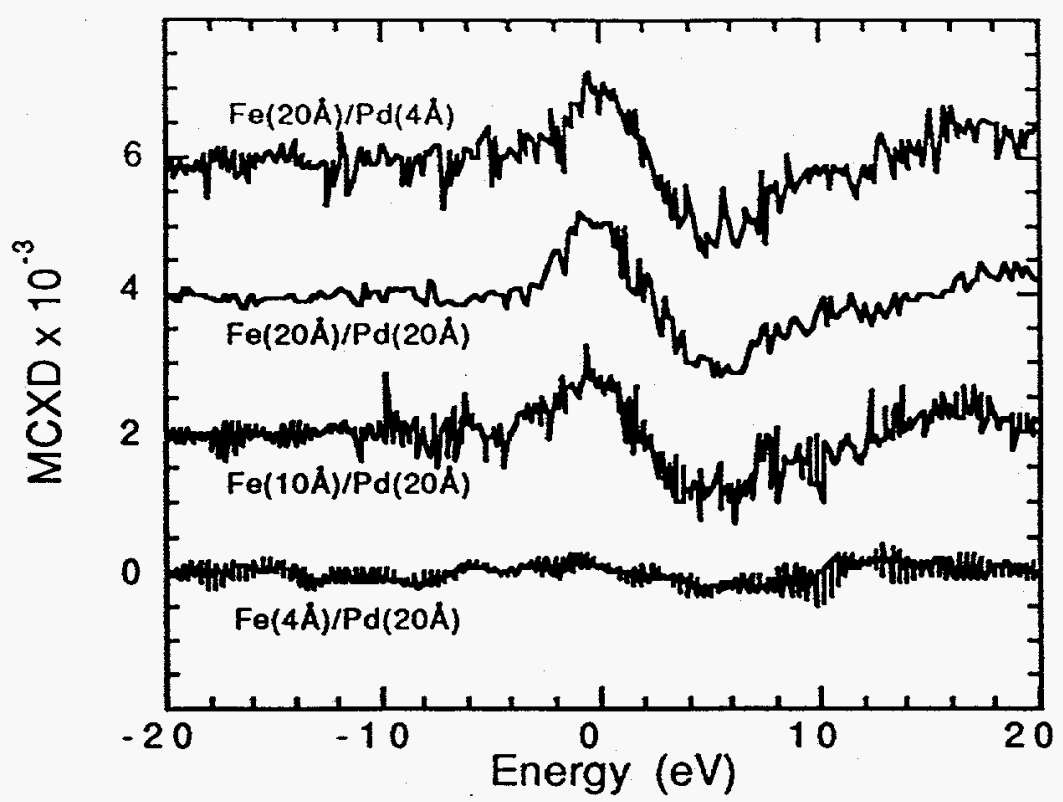

Figure 5. Fe K-edge MCXD spectra of Fe/Pd multilayers taken at room temperature. The energy axis is scaled to the absorption edge positions and the MCXD signals are normalized to the absorption edge step height. The spectra have been shifted vertically with respect to $\mathrm{Fe}(4 \AA) / \mathrm{Pd}(20 \AA)$.

The room-temperature MCXD signals of the Fe K-edge (Fig. 5) in these samples are the same shape and show an increase in amplitude as the Fe layer thickness increases. The amplitudes of $\mathrm{Fe}(20 \AA) / \mathrm{Pd}(20 \AA)$ and $\mathrm{Fe}(20 \AA) / \mathrm{Pd}(4 \AA)$ are almost identical and smaller than that of $\mathrm{Fe}$ foil. This suggests that the overall magnetic properties of $\mathrm{Fe}$ in the systems where $\mathrm{Fe}$ is held constant and Pd is varied are similar to that of the bulk iron. For $\mathrm{Fe} / \mathrm{Co}$ multilayer and alloy systems Pizzini, et al., [12] note a change of shape and amplitude of the negative MCXD peak and suggest that it may be correlated to the magnetic moment of the absorbing atom. However, we see little or no change in the shape of the negative peak as compared to the positive peak in the $\mathrm{Fe} / \mathrm{Pd}$ multilayer systems, indicating that the $\mathrm{Pd}$ is not contributing very much at room temperature.

From the magnetometry results in Fig. 2, it can be noted that at room temperature, the saturation magnetization $\left(\mathrm{M}_{\mathrm{S}}\right)$ for the multilayers is relatively small compared to the low temperature values and also that they are close to the Fe bulk $M_{S}$ value. This suggests that the enhanced $M_{S}$ values of these systems might manifest themselves in the MCXD measurements performed at low rather than room temperatures. In other words, we would be more likely to see the $\mathrm{Pd}$ contribution to the $\mathrm{M}_{\mathrm{S}}$ value at low temperatures, rather than at higher or room temperatures.

In conclusion; the structural information from XAS measurements indicates that for systems where the Pd is held constant and $\mathrm{Fe}$ is varied, the Fe layers become more densely packed (less bcc like) as the Fe layer thickness is decreased. This suggests the formation of an ordered FePd compound at the interface which can have an fcc or fct structure.

At low temperatures, at the interface, $\mathrm{Pd}$ atoms are polarized by the Fe layer. The enhancement at $5 \mathrm{~K}$ corresponds to a moment of $\approx 2.5 \mu_{\mathrm{B}}$ per interface $\mathrm{Pd}$ atom. The polarized moment measured at $4.2 \mathrm{~K}$ for fixed Fe thickness is nearly independent of Pd thickness, indicating a majority of the polarized moment is localized at the Fe/Pd interface. The M-vs.-T data is represented by $\mathrm{T}^{3 / 2}$-behavior expected for magnons in a 3-D ferromagnetic. From this, we infer that the Fe layers are ferromagnetically coupled through the Pd layers giving rise to the 3-D temperature behavior. For fixed Fe thickness the saturation magnetization is strongly temperature dependent for thicker Pd layers as indicated in Figure 2. At room temperature, the SQUID and the MCXD indicate that the Pd contribution to the $\mathrm{M}_{S}$ is small. 
Fermanent address: Department of Physics, Northern Illinois University, DeKalb, IL 60115 and Materials Science Division, Argonne National Laboratory, 9700 S. Cass, Argonne, IL 60439.

\section{ACKNOWLEDGMENTS}

SMM would like to thank the National Science Foundation for support under grant number INT-9304567 and Northern Illinois University, State of Illinois under the HECA Grant. The work at Argonne National Laboratory was supported by US DOE-BES Materials Sciences under contract \#W-31-109-ENG-38.

\section{REFERENCES}

1. J. Crangle and W.R. Scott, J. Appl. Phys. 36, 921 (1965). Also see G. J. Nieuwenhuys, Adv. Phys. 24, 515 (1975).

2. E. E. Fullerton, D. Stoeffler, K. Ounadjela, B. Heinrich, Z. Celinski, J. A. C. Bland, Phys. Rev. B, in press (1994).

3. J.R. Childress, A. Schuhl, J.-M. George, O. Durand, P. Galtier, V. Cros, K. Ounadjela, R. Kergoat and A. Fert, Magnetism and Structure in Systems of Reduced Dimension, (Plenum, New York, 1993); A. Schuhl, J.R. Childress, J.M. George, Pl.Galtier, O.Durand, A. Barthelemy and A. Fert, J. Magn. Magn. Mat. 121, 275 (1993).

4. Z. Celinski, B. Heinrich, J.F. Cochran, W.B. Muir, A.S. Arrott, J. Kirschner, Phys. Rev. Lett. 65,1156 (1990); K. Kyuno, R. Yamamoto, and S. Asano, J. Magn. Magn. Mat. 126, 239 (1993); B. Hillebrands, P. Baumgart, and G. Güntherodt, Appl. Phys. A 49, 589 (1989); A. Boufelfel, R. M. Emrick, and C. M. Falco, Phys. Rev. B 43, 13152 (1991); J. Quinn, Y.S. Li, H. Li, D. Tian, F. Jona, P. M. Marcus, Phys. Rev. B 43, 3959 (1991), S.M. Zhou, L.Y. Chen, Y. Su, X.W. Feng, Y.H. Qian, and X.D. Xie, J. Magn. Magn. Mat. 128, 391 (1993)

5. For example, see T.M. Hayes and J.B. Boyce, Solid State Physics, Vol. 37, 173 (1982) and S. M. Heald in X-ray Absorption, ed. D.C. Koningsberger and R. Prins (Chemical Analysis 92, 1984, John Wiley \& Sons) pp. 119-161.

6. G. van der Laan, B.T. Thole, G.A. Sawatzky, J.B. Goedkoop, J.C. Fuggle, J.M. Esteva, R. Karnatak, J.P. Remeika, H.A. Dabkowska, Phys. Rev. B 34, 6529 (1986).

7. G. Schütz, W. Wagner, W. Wilhem, P. Kienle, R. Zeller, R. Fraham, G. Materlik, Phys. Rev. Lett. 58, 737 (1987), and S. Stähler, G. Schütz, H. Ebert, Phys. Rev. B 47, 818 (1993).

8. J. Goedkoop, PhD thesis, University of Nijmegen, 1989.

9. H. Ebert, P. Strange, B.L. Gyorffy, J. Appl. Phys. 63, 3055 (1988), X. Wang, T.C. Leung, B.N. Harmon, P. Carra, Phys. Rev. B 47, 9087 (1993), and T. Jo and S. Imada, J. Phys. Soc. Jpn. 62, 3721 (1993).

10. E. E. Fullerton, I. K. Schuller, H. Vanderstraeten, and Y. Bruynseraede, Phys. Rev. B 45, 9292 (1992).

11. E. E. Fullerton, I. K. Schuller, F. T. Parker, K. Svinarich, G. Eesley, R. Bhadra, and M Grimsditch, J. Appl. Phys. 73, 7370 (1993).

12. S. Pizzini, A. Fontaine, E. Dartyge, C. Giorgetti, F. Baudelet, J.P. Kappler, P. Boher and F. Giron, Phys. Rev B 50, 3779 (1994). Also, for Ni-Fe alloys, see H. Sakurai, F. Itoh, H. Marumaya, A. Koizumi, K. Kobayashi, H. Yamazaki, Y. Tanji, and H. Kawata, J. Phys. Soc. Jpn. 62, 459 (1993). 